\title{
Engineered Coatings via the Assembly of Amino-Quinone Networks
}

\author{
Qi-Zhi Zhong, Joseph J. Richardson, Ai He, Tian Zheng, René Lafleur, Jianhua Li,
} Wen-Ze Qiu, Denzil Furtado, Shuaijun Pan, Zhi-Kang Xu, Ling-Shu Wan* and Frank Caruso*

\author{
[a] Q.-Z. Zhong, A. He, Dr. W.-Z. Qiu, Prof. Z.-K. Xu, Prof. L.-S. Wan \\ MOE Key Laboratory of Macromolecular Synthesis and Functionalization, Key Laboratory of Adsorption and Separation Materials \& Technologies of \\ Zhejiang Province, Department of Polymer Science and Engineering \\ Zhejiang University, Hangzhou 310027 (China) \\ E-mail: Iswan@zju.edu.cn \\ [b] Q.-Z. Zhong, Dr. J. J. Richardson, Dr. T. Zheng, Dr. R. Lafleur, Dr. J. Li, D. Furtado, Dr. S. Pan, Prof. F. Caruso \\ ARC Centre of Excellence in Convergent Bio-Nano Science and Technology, and the Department of Chemical Engineering \\ The University of Melbourne \\ Parkville, Victoria 3010 (Australia) \\ E-mail: fcaruso@unimelb.edu.au \\ Supporting information for this article is given via a link at the end of the document.
}

\begin{abstract}
Engineering coatings with precise physicochemical properties allows for control over the interface of a material and its interactions with the surrounding environment. However, assembling coatings with well-defined properties on different material classes remains a challenge. Herein, we report a co-assembly strategy to precisely control the structure and properties (e.g., thickness, adhesion, wettability, and surface potential) of coatings on various materials (27 substrates examined) using quinone and polyamine building blocks. By increasing the length of the amine building blocks from small molecule diamines to branched amine polymers, we can tune the properties of the films, including the thickness (from $\sim 5$ to $\sim 50 \mathrm{~nm}$ ), interfacial adhesion ( 0.05 to $5.54 \mathrm{nN}$ ), water contact angle $\left(130\right.$ to $\left.40^{\circ}\right)$, and surface potential $(-42$ to $28 \mathrm{mV})$. The films can be post-functionalized through the in situ formation of diverse nanostructures, including nanoparticles, nanorods, and nanocrystals. Our approach provides a platform for the rational design of engineered, substrate-independent coatings for various applications.
\end{abstract}

\section{INTRODUCTION}

Engineering functional coatings is important for fundamental investigations into interfacial phenomena, ${ }^{[1-3]}$ as well as for practical applications in areas including lithography, ${ }^{[4,5]}$ superwettability, ${ }^{[6,7]}$ separation, ${ }^{[8,9]}$ encapsulation, ${ }^{[10]}$ catalysis, ${ }^{[11]}$ and bioimaging. ${ }^{[12]} \mathrm{A}$ variety of methods have been developed to coat specific substrates and endow them with desired properties, including self-assembled monolayer (SAM) formation, ${ }^{[13]}$ electrochemical deposition, ${ }^{[14]}$ and layer-by-layer assembly. ${ }^{[15-17]}$ Alternatively, substrate-independent functional thin films such as organic polydopamine (PDA) coatings ${ }^{[18]}$ and hybrid metalphenolic networks (MPNs), ${ }^{[19]}$ have attracted widespread interest owing to their wide-ranging adhesion characteristics, ${ }^{[20]}$ high antioxidation properties, ${ }^{[21]}$ and high stability, ${ }^{[22]}$ which are all imparted by the catechol-containing building blocks in the coatings. Although current substrate-independent films show potential for biomedicine, ${ }^{[23]}$ separation processes, ${ }^{[24]}$ and catalysis, ${ }^{[25}$ it is still challenging to predictably engineer a broad range of properties (e.g., charge, thickness, wettability, adhesion, transparency) of coatings. ${ }^{[26]}$

Herein, we present a co-assembly strategy for tuning the structures and properties of coatings on diverse substrates using a library of quinones and polyamines. This approach leverages the ability of both quinones and amines to disrupt the hydration layer around different substrates (e.g., polymer, metal, and mineral), thereby ensuring adhesion of the resulting coatings. By varying the type of the quinone and polyamine building blocks, the interfacial adhesion, film structure, wettability, surface potential, and transparency can all be tailored. Furthermore, the resulting coatings can mediate different secondary reactions, including the liquid-phase epitaxy of crystals, to synthesize various nanomaterials with controllable size and density on the surface of the coatings.

The challenges associated with engineering substrateindependent coatings derive primarily from the solvents used for synthesis, which can form tight solvation layers around the substrates, thereby preventing the adhesion and cross-linking of the coating building blocks on the surface. ${ }^{[27-29]}$ In nature, to achieve adhesion in aqueous environments, animals (e.g., mussels, barnacles, and spiders) can produce proteins with positively charged residues that displace the interfacial hydration layer from wet surfaces, ${ }^{[30-32]}$ followed by anchoring of the proteins both to the substrate (through catechol residues) and to other proteins (via catechol-amine cross-linking). Inspired by these properties, we envision that a two-component system comprising polyamines and quinones that can displace the hydration layer around a substrate to achieve adhesion, followed by extensive cross-linking to form substrate-independent, robust coatings (Figure 1a). Specifically, the positively charged amino groups in the polyamines facilitate the rapid removal of hydration layers surrounding mineral surfaces, while the quinones display noncovalent interactions (e.g., $\pi-\pi$ stacking) and metal 


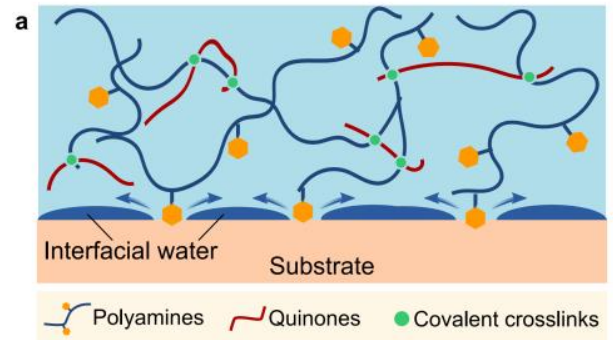<smiles>Nc1ccc(O)c2c1C=CC(=O)C2=O</smiles>

c
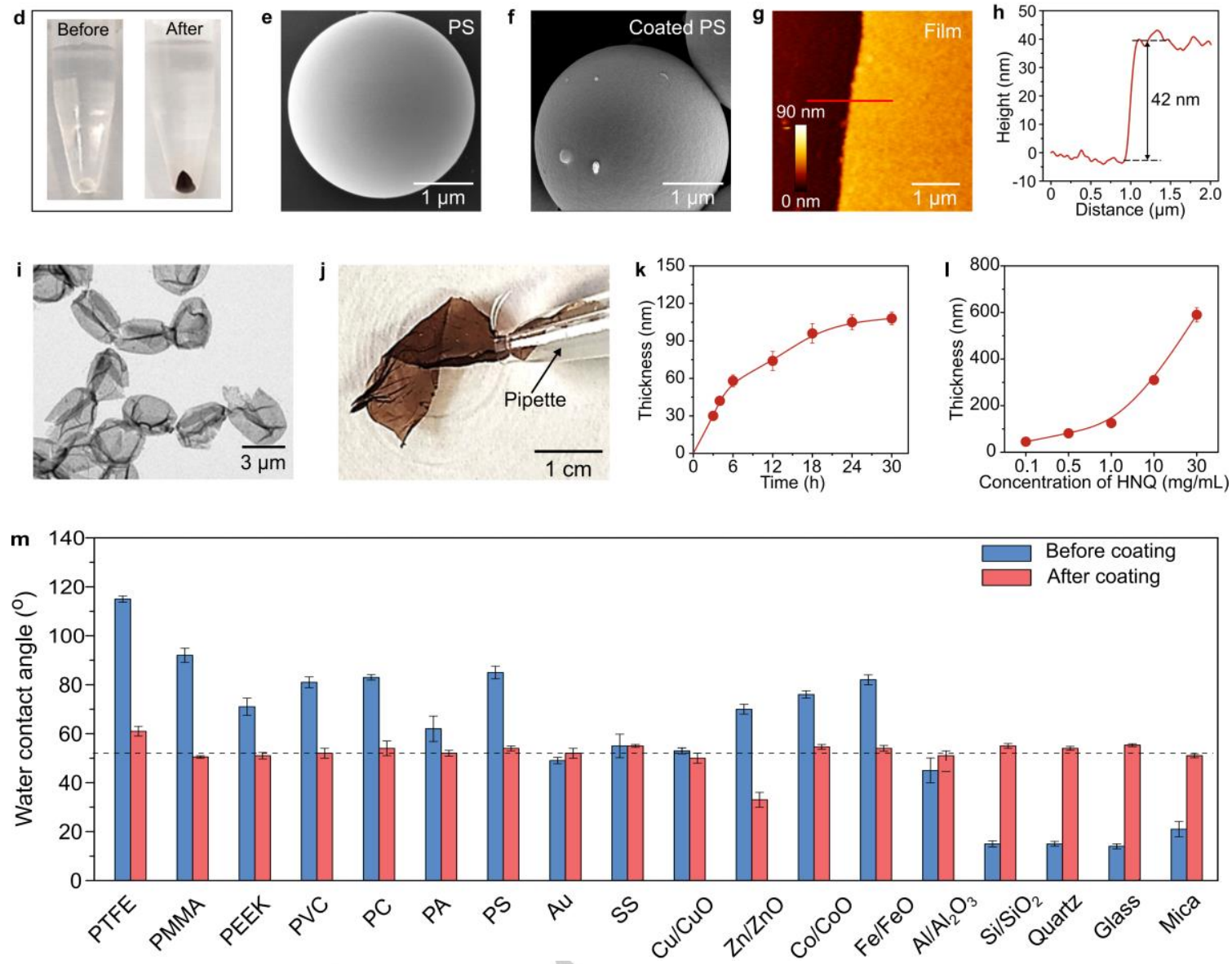

Figure 1. HNQ-PEI formation on surfaces. a) Proposed mechanism for AQN formation that integrates the displacement of interfacial water by amino groups for adhesion of quinone and polyamine and their subsequent cross-linking via covalent reactions. b,c) Molecular structures of $H N Q(b)$ and polyamine (PEI) (c). $d-f$ ) Photographs of PS particles in solution (d) and SEM images of a PS particle before (e) and after (f) coating. g,h) AFM image (g) showing HNQ-PEI coating on a $\mathrm{Si} / \mathrm{SiO}_{2}$ surface and the corresponding thickness profile (h). i,j) TEM image of HNQ-PEI capsules (i) and photograph of a free-standing film (j). $\left.k, \mathrm{l}\right)$ Thickness growth of a HNQ-PEI film as a function of time $(\mathrm{k})$ and concentration of $\mathrm{HNQ}$ ( $x$-axis is not drawn to scale) (l) measured by ellipsometry. $\mathrm{m}$ ) Water contact angles of different planar substrates before and after HNQ-PEI coating.

coordination on polymer and metal oxide surfaces. Compared with catechols, quinones exhibit a lower degree of adhesion to the substrates, ${ }^{[33]}$ thus allowing for a higher degree of control over the coating deposition kinetics and therefore the amount deposited; consequently, the coating/film properties can be readily tuned. Following adhesion, the quinones and polyamines extensively cross-link to form stable amino-quinone networks (AQNs) within a few minutes. The present study expands the toolbox of substrate-independent coatings while providing new insight into their structure-property relationships.

\section{RESULTS AND DISCUSSION}

\section{Assembly of AQN coatings}

We first examined the formation of AQNs using 5-hydroxy-1,4naphthoquinone (HNQ) (Figure 1b) and poly(ethyleneimine) (PEI, $M_{\mathrm{w}} \sim 600 \mathrm{Da}$ ) (Figure 1c) as a model quinone/polyamine pair on spherical (Figure 1d-f), columnar (Figure S1), and planar (Figure $1 \mathrm{~g}, \mathrm{~h}$ ) substrates. HNQ was chosen as a model quinone owing to its relatively simple structure and its promising inherent antitumor and anti-bacterial properties. ${ }^{[34,35]} \mathrm{HNQ}$ is known to couple covalently with amines via Michael addition and Schiff base reactions, ${ }^{[36]}$ which was likewise observed herein from the electrospray ionization-mass spectrometry and UV-visible (UVvis) spectroscopy analyses of the HNQ-PEI solutions (Figure S2). The proposed network structure is shown in Figure S3. Upon 
coating of $\mathrm{HNQ}-\mathrm{PEI}$ on polystyrene (PS) particles, the particle surface turned red (Figure 1d). The formation of conformal HNQPEI coatings on the different substrates examined (i.e., PS particles, stainless steel mesh, and $\mathrm{Si} / \mathrm{SiO}_{2}$ substrates) was confirmed by scanning electron microscopy (SEM) and atomic force microscopy (AFM) measurements, which showed a film with a thickness of $\sim 40 \mathrm{~nm}$ and root-mean-square roughness of $\sim 2 \mathrm{~nm}$ on $\mathrm{Si} / \mathrm{SiO}_{2}$ surface after coating for $4 \mathrm{~h}$ (Figure 1g,h). Xray photoelectron spectroscopy (XPS), Fourier transform infrared spectroscopy (FTIR), and energy-dispersive X-ray spectroscopy (EDS) analyses indicated the homogenous distribution of $\mathrm{HNQ}$ and PEI on the coated surfaces (Figure S4).

To evaluate the applicability of the HNQ-PEI films to different substrates, a HNQ/PEI mixture was used to coat a range of substrates (27 substrates examined) with various shape and composition, including polymers (polytetrafluoroethylene (PTFE), poly(methyl methacrylate) (PMMA), polyetheretherketone (PEEK), polyvinyl chloride (PVC), polycarbonate (PC), polyamide (PA), PS, poly(lactic-co-glycolic acid), melamine formaldehyde, and polydimethylsiloxane), a noble metal (Au), metal plates with a native oxide surface (stainless steel (SS), $\left.\mathrm{Cu} / \mathrm{CuO}, \mathrm{Zn} / \mathrm{ZnO}, \mathrm{Co} / \mathrm{CoO}, \mathrm{Fe} / \mathrm{FeO}, \mathrm{Al} / \mathrm{Al}_{2} \mathrm{O}_{3}\right)$, minerals $\left(\mathrm{Si} / \mathrm{SiO}_{2}\right.$, quartz, and mica), and ceramics (glass and calcium carbonate $\left(\mathrm{CaCO}_{3}\right)$ ), see Table $\mathrm{S} 1$. All studied substrates turned red following coating (Figure S5). In addition, after coating, the average water contact angles and zeta-potential values of almost all studied substrates changed to around $55^{\circ}$ and $28 \mathrm{mV}$, respectively (Figure $1 \mathrm{~m}$, and Figures S6 and S7), indicating an effective coating process irrespective of the substrate. The lower water contact angle of $\mathrm{Zn} / \mathrm{ZnO}$ was possibly due to the increase in surface roughness by etching the substrates through the coordination reaction between $\mathrm{HNQ}$ and $\mathrm{Zn} / \mathrm{ZnO}$ in solution ${ }^{[6]}$. Furthermore, HNQ-PEI capsules (i.e., hollow free-standing spherical films) (Figure 1i) and macroscopic free-standing flexible films (Figure 1j) were obtained upon removal of the template substrates; these free-standing films have potential uses in, for example, advanced separation, ${ }^{[37]}$ drug delivery ${ }^{[38]}$ and microreactions. ${ }^{[19]}$ These results demonstrate that the HNQPEI films can be used to coat various substrates in aqueous solutions and can be processed as separate (free-standing) materials for specific applications.

The influence of deposition conditions on film thickness and morphology was also examined. Ellipsometry and AFM measurements showed that the HNQ-PEI film thickness varied as a function of film growth time and reached $\sim 100 \mathrm{~nm}$ after 24 $\mathrm{h}$ (Figure $1 \mathrm{k}$ and Figure S8). In addition, HNQ-PEI film formation successfully occurred in an anaerobic environment, saline solutions with high ionic strength (e.g., $1 \mathrm{M} \mathrm{NaCl}$ ), aqueous solutions of $\mathrm{pH} 4-12$, and various organic solvents (Figure S9), including ethanol $(\mathrm{EtOH})$, methanol, acetonitrile $(\mathrm{ACN})$, tetrahydrofuran (THF), and dimethylformamide (DMF). Furthermore, film growth was concentration-dependent (the building block weight ratio was kept at 1:1, Figure 11). For instance, the thickness reached $\sim 300 \mathrm{~nm}$ when the concentration of both $\mathrm{HNQ}$ and PEI was $10 \mathrm{mg} / \mathrm{mL}$ at $\mathrm{pH} 10$ (Figure 11, Figure S10). These results collectively demonstrate the high controllability of the film growth.

\section{Formation mechanism of AQN coatings}

When wet, minerals such as $\mathrm{Si} / \mathrm{SiO}_{2}$, mica, and quartz are difficult to coat with organic materials owing to considerably stronger hydration barriers ${ }^{[27]}$ present than under dry conditions. Therefore, the formation of the HNQ-PEI coatings was investigated on a wet surface using silicon that had been exposed to ambient air $\left(\mathrm{Si} / \mathrm{SiO}_{2}\right)$ as a model substrate. Coating of the mineral surfaces, i.e., $\mathrm{Si} / \mathrm{SiO}_{2}$, quartz, glass, and mica was unsuccessful when solely using $H N Q$, which confirmed the unfavorable interactions between $\mathrm{HNQ}$ and the studied minerals (Figures S11 and S12). However, adding a small amount of PEI $\left(1 \times 10^{-4} \mathrm{mg} / \mathrm{mL}, 160 \mathrm{nM}\right)$ enabled the formation of HNQ-PEI films with a thickness of $\sim 12 \mathrm{~nm}$ on $\mathrm{Si} / \mathrm{SiO}_{2}$ surfaces (Figure S13), indicating the importance of PEI for film formation. We note that $\mathrm{HNQ}$ by itself could form particles (possible due to the selfpolymerization of $\mathrm{HNQ}$ as previously reported ${ }^{[39]}$ ) and adhere to polymer, noble metal, and metal oxide surfaces, without the need of $\mathrm{PEI}$, owing to the hydrophobic and chelation interactions of HNQ (see the discussion in Figures S14-S16).

To understand the role of PEl in enabling coating on wet surfaces, colloidal-probe AFM was used to measure the forces that the quinone and polyamine building blocks generate toward $\mathrm{SiO}_{2}$. An approach-retraction loop ${ }^{[40]}$ provided information about the interactions upon approach and retraction of $\mathrm{a} \mathrm{SiO}_{2}$ particle from a HNQ-coated PS surface. During approach (Figure 2a), repulsion was observed, and the attraction between the HNQ coating and silica surface was negligible when they were separated (retraction). In contrast, with the addition of PEI $\left(\sim 10^{-4}\right.$ $\mathrm{mg} / \mathrm{mL}$ ) to the water, the HNQ coating was rapidly $(<5 \mathrm{~min})$ attracted to the silica particle (Figure $2 \mathrm{~b}$ ) with a high adhesion energy $\left(E_{\mathrm{ad}}\right)$ of $6.6 \times 10^{-2} \mathrm{~mJ} / \mathrm{m}^{2}$, which was evaluated using the Johnson-Kendall-Roberts model: ${ }^{[41]}$

$$
E_{\mathrm{ad}}=\frac{F_{\mathrm{ad}}}{1.5 \pi R}
$$

where $F_{\text {ad }}$ is the adhesion force between the colloidal particle and the substrate surface, and $R$ is the radius of the colloidal probe. The high adhesion energy obtained confirmed the essential role of PEI in the formation of HNQ-PEI coatings, which is consistent with a previous study that showed that amino groups can displace hydration layers on wet surfaces to promote adhesion. ${ }^{[27,28]}$

To confirm the role of the amino groups in film formation, a freestanding HNQ-PEI film was prepared using an etchable silica substrate (Figure S17). XPS analysis demonstrates that the bottom of the HNQ-PEI film (the side attached to the substrate) featured a higher N/O ratio (i.e., 0.49) than the top of the film $(\mathrm{N} / \mathrm{O}$ ratio $=0.38)$ (Figure $2 \mathrm{c})$, suggesting a higher amount of $\mathrm{PEI}$ and a lower amount of $\mathrm{HNQ}$ closer to the substrate. In the absence of $H N Q$, PEI only formed an adsorption layer with a thickness of less than $2 \mathrm{~nm}$ even after film growth for $24 \mathrm{~h}$ and no free-standing films were obtained. These results support the ability of quinones and polyamines to interact with different substrates, as well as the synergetic cross-linking to generate thick (>10 nm) films (see the discussion in Figure S18).

The strong adhesion to substrates at the nanoscale endows the HNQ-PEI coatings with excellent mechanical stability at the macroscopic scale. The HNQ-PEI coatings withstood peeling by Scotch $^{\mathrm{TM}}$ tape, sonication, and washing, with negligible changes in the water contact angle and optical transmittance (Figure S19). Furthermore, the HNQ-PEI coatings were chemically stable in 


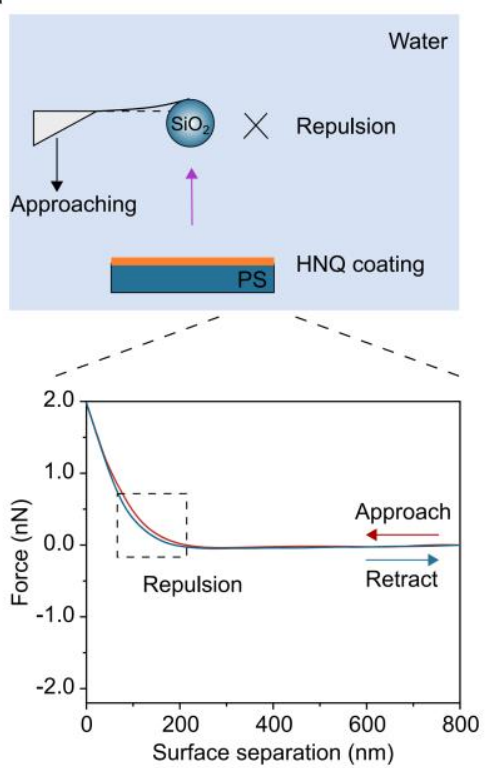

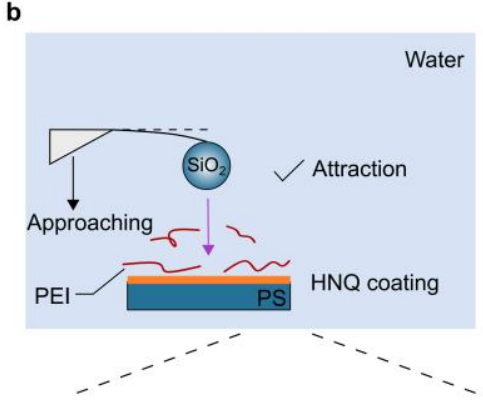

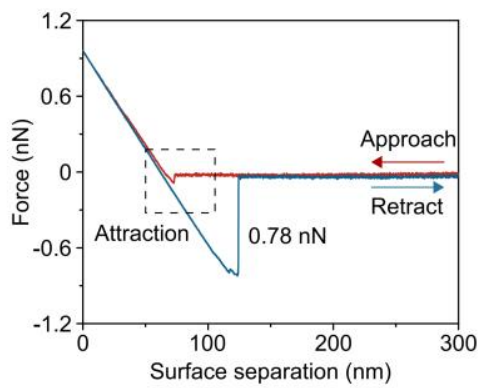

Distribution of $\mathrm{PEI}$ and $\mathrm{HNQ}$ in film

$\mathrm{PEI} / \mathrm{HNQ}$ ratio
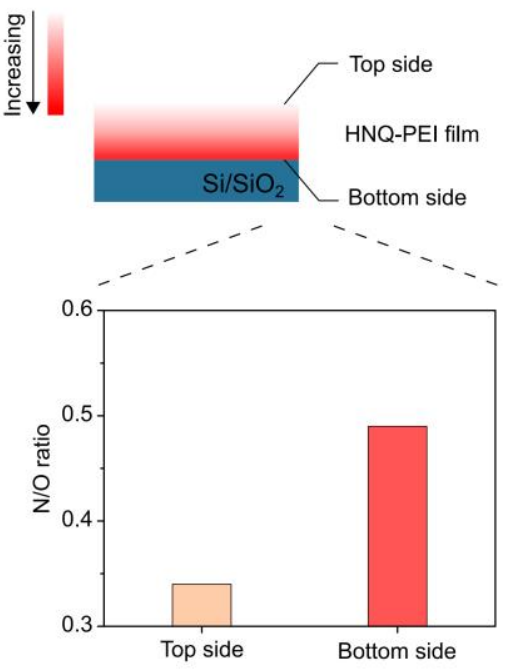

d
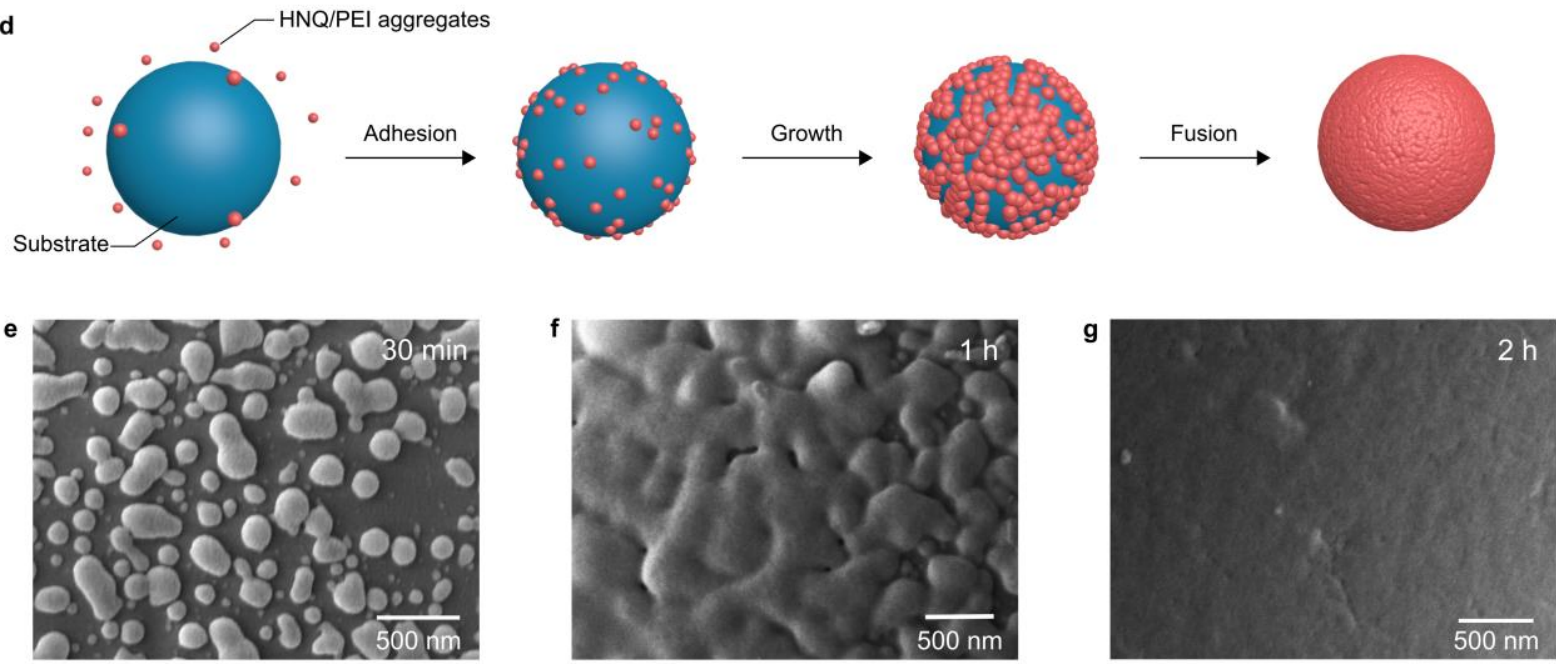

Figure 2. Analysis of adhesion force, building block distribution, and film formation. a,b) Schematic and representative force-distance curves of a SiO ${ }_{2}$-attached AFM probe approaching a PS substrate with a HNQ coating in water before (a) and after (b) the addition of PEl. c) Schematic and XPS analysis of the elemental distribution on the top and bottom sides of a HNQ-PEI film prepared on a Si/SiO ${ }_{2}$ substrate. $d$ ) Schematic of the formation of $\mathrm{HNQ}-\mathrm{PEI}$ coating on a substrate. e-g) SEM images showing the morphology of HNQ-PEI coating during film growth.

various organic solvents (e.g., DMF, ACN, EtOH, isopropanol, acetone, THF, ethyl acetate, toluene, and $n$-hexane), as indicated from the relatively constant water contact angles of the coated surfaces following incubation in the solvents for $12 \mathrm{~h}$ (Figure S20). The HNQ-PEI coatings were also stable in aqueous solutions of $\mathrm{pH}$ between 1 and 12 (Figure S20). However, a reduction $(\sim 60 \%)$ in the film thickness on a PS surface was noted following incubation in aqueous solution at $\mathrm{pH}$ 14 , which may be due to the hydrolysis of the imine bond in $\mathrm{HNQ}$ PEI.[22] Unlike catechol-based coatings whose formation and stability are readily influenced by oxidants, $\mathrm{pH}$, and ionic strength, ${ }^{[41]}$ the versatile deposition conditions and high stability of $A Q N s$ provide an attractive toolbox for engineering robust and durable underwater adhesives, water-resistant materials, and substrate-independent coatings in harsh environments.

In addition to the adhesion driving forces, we investigated the coating formation process by monitoring the assemblies at different time points in solution (i.e., in the absence of a substrate) and on a substrate (Figure 2d). TEM analysis revealed that $\mathrm{HNQ}$ and $\mathrm{PEI}$ react to form nanoaggregates in solution (Figure S21). These nanoaggregates then adsorb onto the substrate and grow into larger aggregates, subsequently fusing into a complete film (Figure 2e-g). 


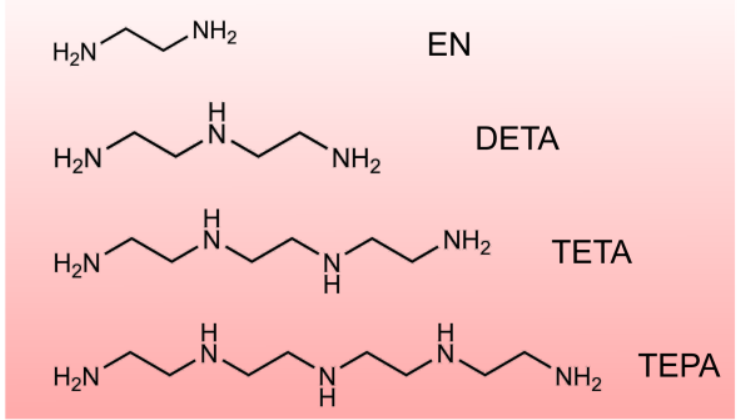

b

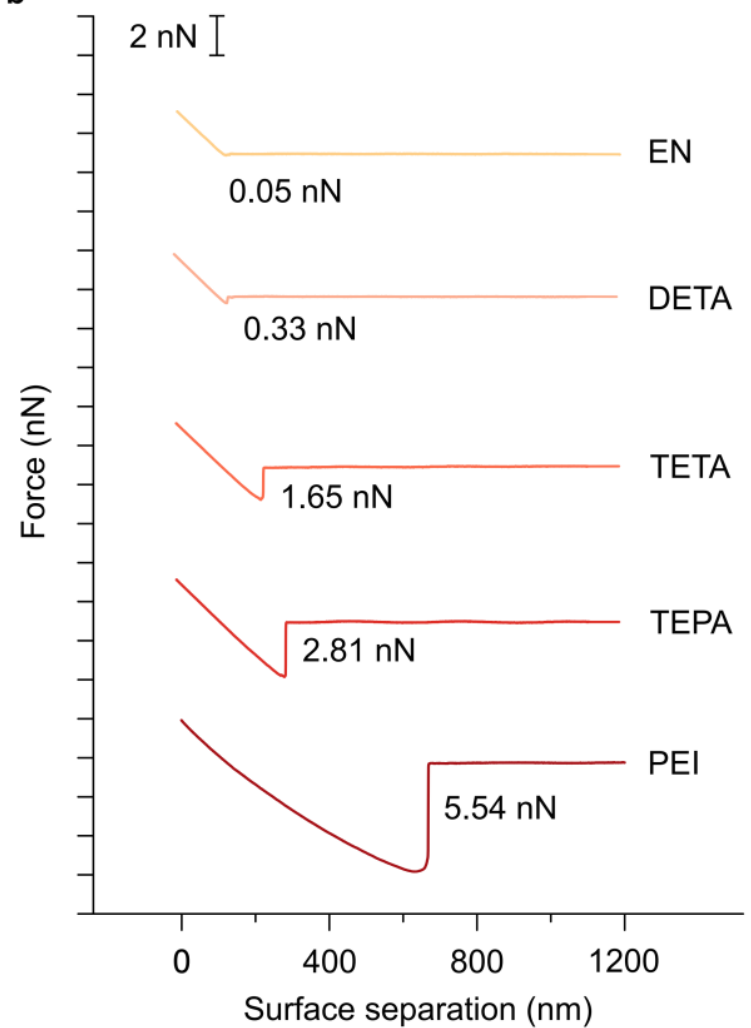

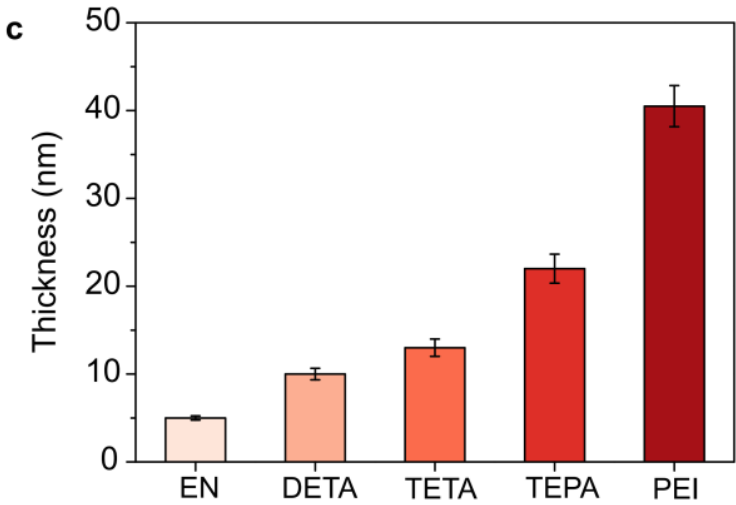

d

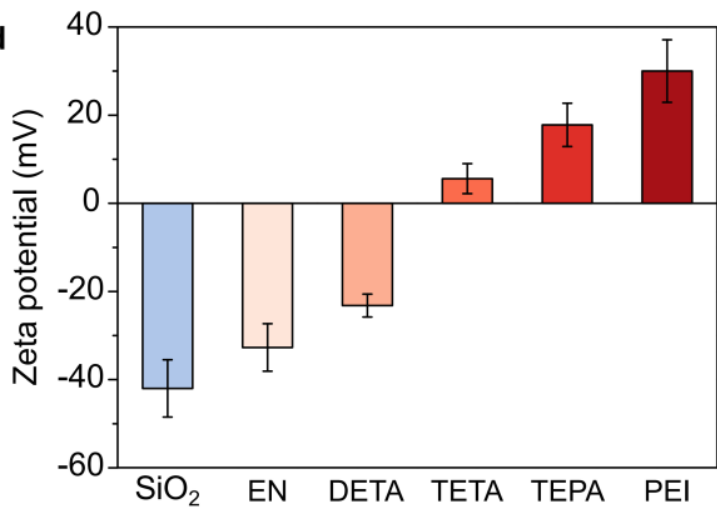

e

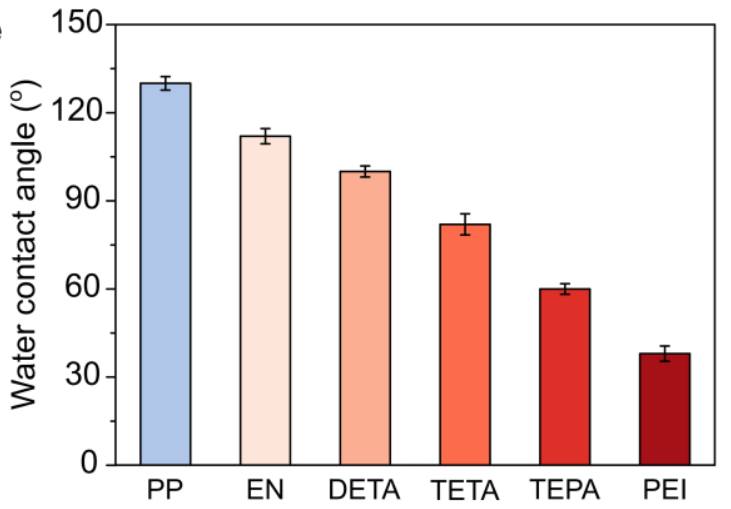

Figure 3. Engineering the properties of $A Q N$ s by varying the polyamine building block at constant concentrations of $\mathrm{HNQ}$ (12 $\mathrm{mM}$ ) and polyamine (2 mM). a) Molecular structures of polyamines. b) Adhesion forces of different AQN coatings (prepared from different polyamines) measured with a SiO ${ }_{2}$-attached AFM probe. c) Thickness of different AQN films. d) Zeta potential values of $\mathrm{SiO}_{2}$ particles before and after coating with different AQNs. e) Water contact angles of PP membranes before and after coating with different AQNs.

\section{Engineering the properties of AQNs}

As amines are one of the most abundant functional groups in synthetic and natural molecules, ${ }^{[42]}$ their structural diversity allows the engineering of AQNs with tunable properties. Accordingly, a variety of AQNs were prepared using different amine-based building blocks (i.e., ethylenediamine (EN), diethylenetriamine (DETA), triethylenetetramine (TETA), and tetraethylenepentamine (TEPA)) and their properties were examined. Increasing the number of amino groups per molecule (the final concentrations of $\mathrm{HNQ}$ and the polyamine were 12 and $2 \mathrm{mM}$, respectively) (Figure $3 \mathrm{a}$ ) led to an increase in the adhesion force from $0.05 \mathrm{nN}$ for $\mathrm{HNQ}-\mathrm{EN}$ (the smallest polyamine studied) to $5.54 \mathrm{nN}$ for HNQ-PEI (the largest polyamine studied) (Figure $3 \mathrm{~b}$ and Figure S22). The film thickness (Figure 3c) and optical transmittance (Figure S23) also changed markedly because of the different adhesion and molecular structures. In addition, owing to the positive charge and hydrophilicity of the amine groups, the zeta-potential values of silica particles increased and the water contact angles on polypropylene (PP) membranes decreased with an increase in the number of amine groups (Figure $3 \mathrm{~d}, \mathrm{e}$ ). Such precise control of surface properties by simply varying the building blocks is valuable in itself and allows for the use of the corresponding coatings as a platform to tune various surface-confined reactions. 
a

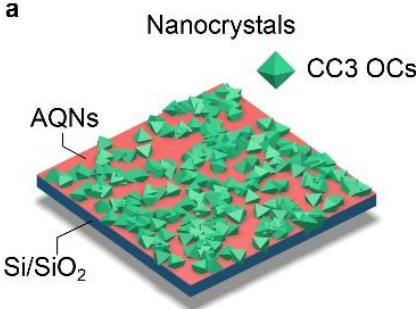

e

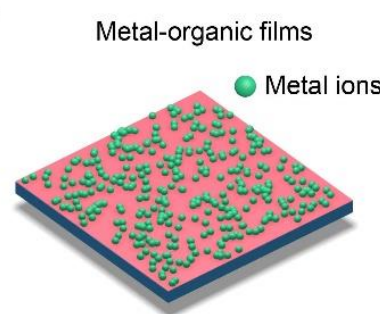

i

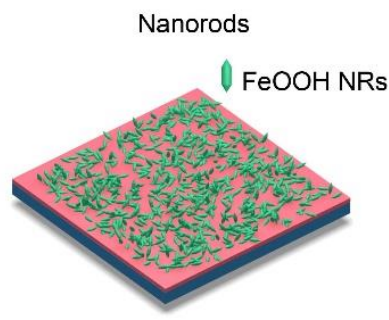

m

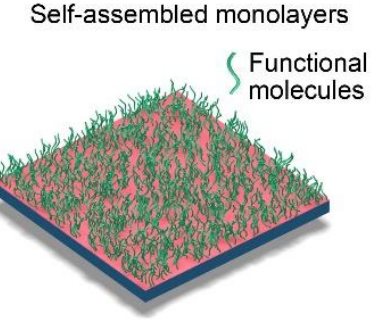

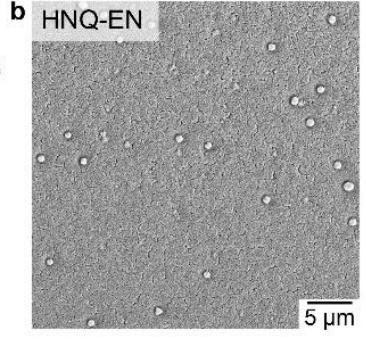
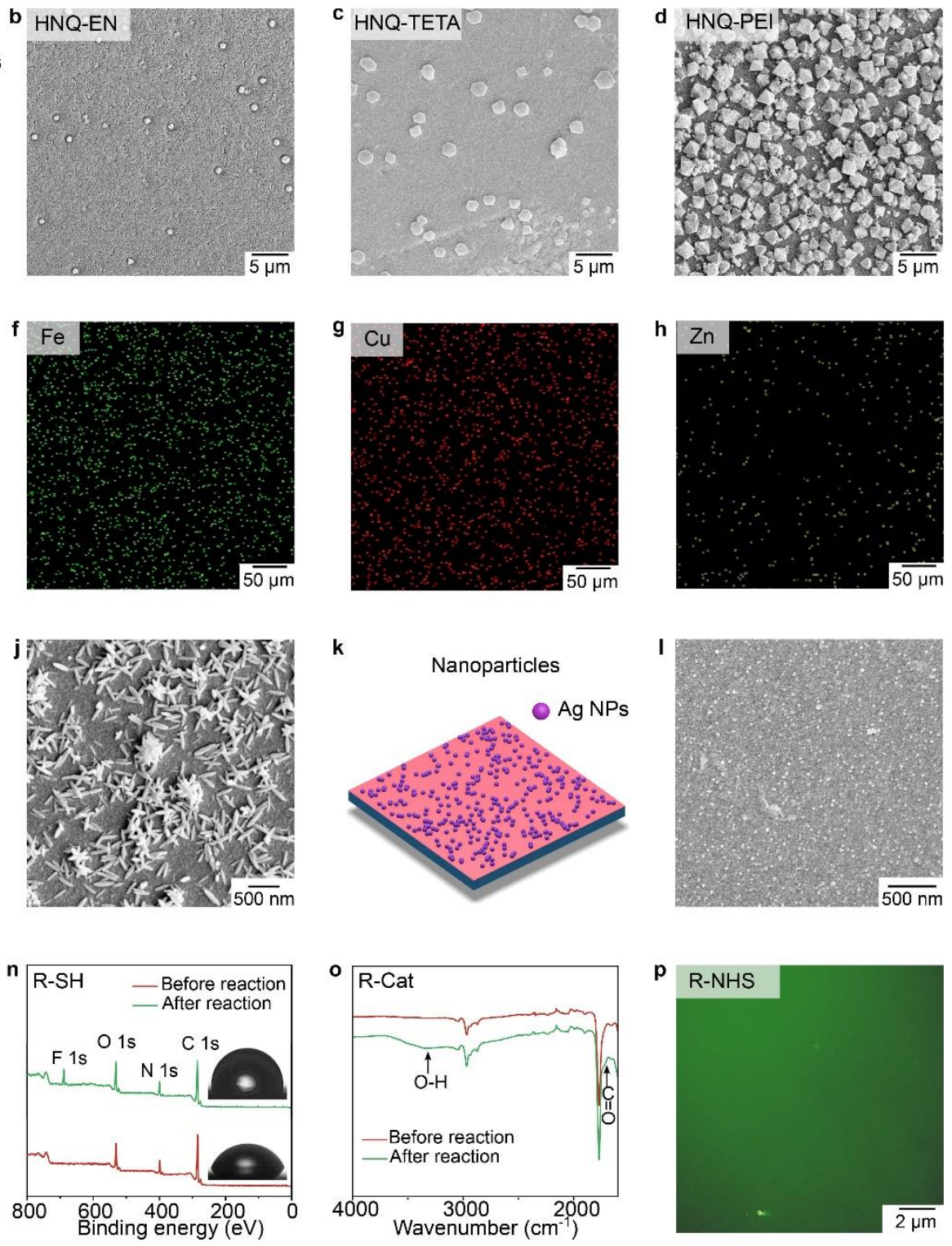

Figure 4. AQN-mediated surface-confined reactions. a-d) Schematic (a) and SEM images of CC3 organic cages (OCs) on HNQ-EN (b), HNQ-TETA (c), and HNQPEI (d) coatings. e-h) Schematic (e) and EDS images of HNQ-PEI coatings chelated with metal ions Fe ${ }^{\prime \prime \prime}(\mathrm{f}), \mathrm{Cu}^{\prime \prime}(\mathrm{g})$, and $\left.\mathrm{Zn}^{\prime \prime}(\mathrm{h}) . \mathrm{i}, \mathrm{j}\right) \mathrm{Schematic}(\mathrm{i})$ and SEM image of mineralization of FeOOH NRs on HNQ-PEI coating (j). k, I) Schematic (k) and SEM image of metallization of Ag NPs on HNQ-PEI coating (I). m-p) Schematic of SAMs $(m)$, and XPS spectra (n) and FTIR spectra (o), and CLSM image (p) of PEI/HNQ coatings conjugated with thiol-containing molecule (R-SH, i.e., PFDT), catechol-containing molecule (R-Cat, i.e., tannic acid), and NHS-containing molecule (R-NHS, i.e., Alexa Fluor ${ }^{\mathrm{TM}} 488 \mathrm{NHS}$ ester), respectively. Insets in (n) are images of water droplets on the corresponding surfaces.

\section{Applications of AQNs}

To demonstrate that the AQN coatings can be used to tune surface-confined reactions, we first explore the effect of surface properties on the formation of nanocrystals. Porous organic cages, a new family of shape-persistent porous crystals, have attracted widespread interest owing to their well-defined pore structures, high crystallinity, and diverse potential applications ${ }^{[43]}$ Such molecular cages are typically synthesized through imine condensation between specific aldehydes and amines. As the amino groups in AQNs can react with aldehydes, they can mediate the crystallization (e.g. nucleation and growth) of porous organic cages on surfaces. ${ }^{[44]}$ Specifically, a typical (1S, 2S)-1,2-cyclohexane diamine cage (CC3) was synthesized on AQN surfaces by liquid-phase epitaxy. By increasing the number of amino groups (two and four per molecule of EN and TETA, respectively, and $\sim 15$ per PEI molecule) on the AQN surfaces, the aldehydes reacted more rapidly and crystallization proceeded more quickly, with a corresponding increase in the CC3 cage size from 0.71 to 1.46 and $1.52 \mu \mathrm{m}$ on HNQ-EN, HNQTETA, and HNQ-PEI surfaces (Figure $4 a-d$ and Figure S24), respectively, after $10 \mathrm{~h}$. The number of CC3 crystals on the films increased from $1.7 \times 10^{10} / \mathrm{m}^{2}$ to $4.1 \times 10^{10} / \mathrm{m}^{2}$ and $2 \times 10^{11} / \mathrm{m}^{2}$ on $H N Q-E N, H N Q-T E T A$, and HNQ-PEI, respectively. This could be attributed to the larger number of reaction sites (i.e., amino groups) for nucleation. ${ }^{[45]}$ The ease of tailorability of the size and density of CC3 cages on surfaces by AQNs can potentially afford modulation of the performance of host-guest systems, porous materials, catenanes, and nanoconfined chemical reactions. ${ }^{[44]}$ 
a

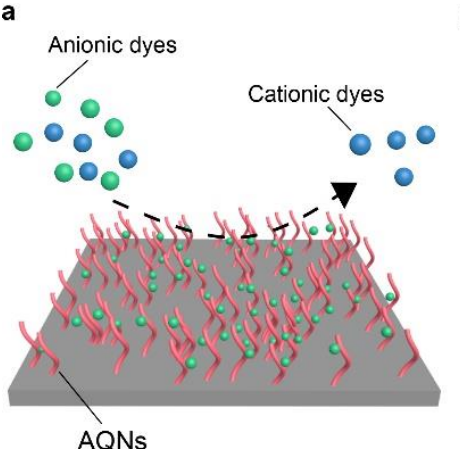

b

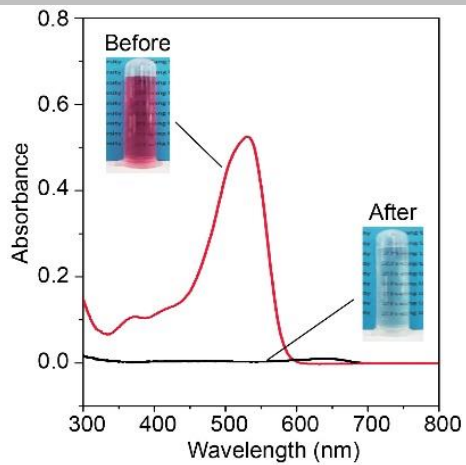

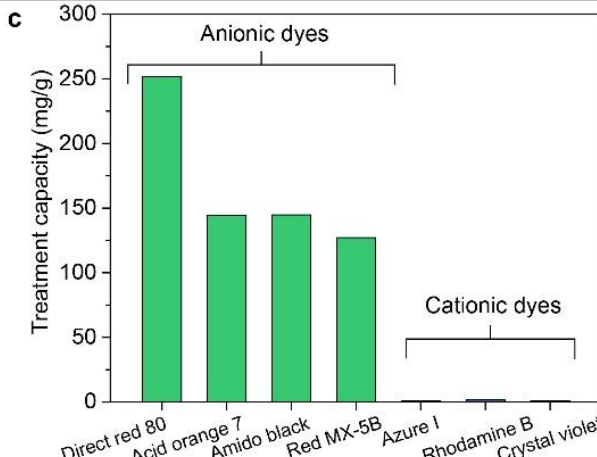

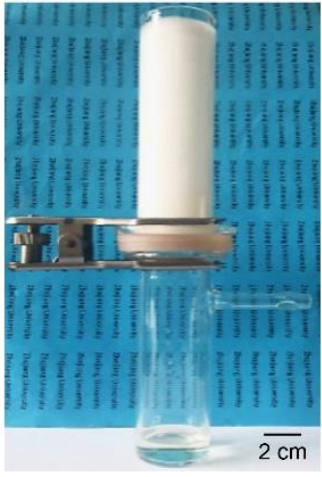

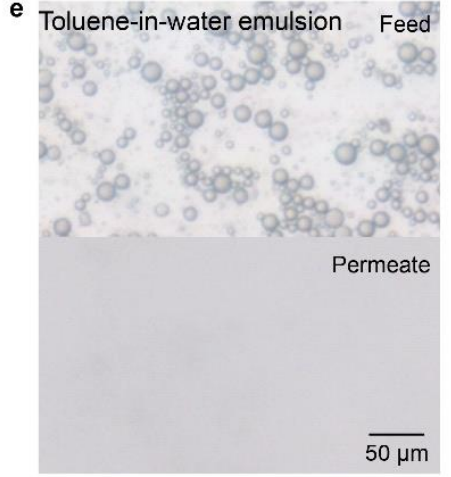

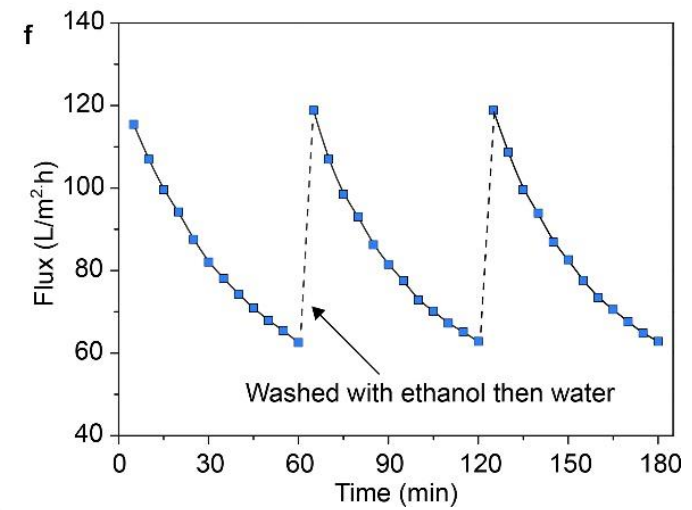

Figure 5. Applications of AQNs for water treatment. a-c) AQNs for organic dyes adsorption: schematic image of an AQN coating for selective dye adsorption (a); UV-vis spectra of the direct red 80 solution before and after treatment (b); and treatment capacity of a series of dye solutions (c). $d-f$ ) AQNs for oil-in-water emulsion separation: filtration unit, built in house, used for the separation of a toluene-in-water emulsion (d); feed and filtrate after treatment (e); and water flux of toluene-inwater separation and cyclic performance of the coated membrane $(\mathrm{f})$.

Besides the liquid-phase epitaxy of organic cages, a range of chemical reactions can occur on AQN coatings. For example, owing to the metal-chelating ability of $\mathrm{HNQ},{ }^{[46]} \mathrm{HNQ}$-PEI coatings can serve as a functional layer to chelate various metal ions (e.g., $\mathrm{Fe}^{\mathrm{III}}, \mathrm{Cu}^{\prime \prime}$, and $\mathrm{Zn}$ ") (Figure $4 \mathrm{e}-\mathrm{h}$ ) to form metal-organic films. Further incubation leads to the formation of $\mathrm{FeOOH}$ nanorods (NRs) (Figure 4i,j) and Ag nanoparticles (NPs) (Figure 4k,l, and Figure S25) on the AQN surfaces through mineralization and metallization processes, respectively. In addition, HNQ-PEI coatings enabled a variety of reactions with organic species to create diverse SAMs (Figure $4 \mathrm{~m}-\mathrm{p}$ ). For instance, thiolcontaining molecules (e.g., $1 \mathrm{H}, 1 \mathrm{H}, 2 \mathrm{H}, 2 \mathrm{H}$-perfluorodecanethiol (PFDT)) formed SAMs through the thiol-adduct reaction with quinones (Figure 4n). ${ }^{[47]}$ Catechol-containing molecules (e.g., tannic acid) complexed with the coating to form a tannic acid layer on the HNQ-PEI surface (Figure 4o). $\mathrm{N}$-hydoxysuccinimide (NHS)-containing molecules (e.g., Alexa Fluor ${ }^{\mathrm{TM}} 488 \mathrm{NHS}$ ester) were conjugated to the coatings by reacting with the amino groups, ${ }^{[48]}$ yielding surfaces with green fluorescence under confocal laser scanning microscopy (CLSM) observation (Figure $4 p)$.

AQNs can also be prepared using different quinone molecules. For example, employing 5,8-dihydroxy-1,4-naphthoquinone or 1,5-dihydroxyanthraquinone both led to successful AQN coatings on substrates (Figure S26). Considering the properties (e.g., color, redox, and bioactivity) of quinones and polyamines, and their ubiquitous presence in nature,${ }^{[49-51]} \mathrm{AQN}$ coatings have the potential to be used for various applications, including organic dye adsorption, oil-in-water separation, and in antibacterial materials ${ }^{[34,35]}$ and organic redox flow batteries. ${ }^{[52]}$ The high zeta potential endowed by the positively charged PEI enabled the use of the coated membrane for selective organic dye adsorption. As shown in Figure $5 \mathrm{a}-\mathrm{c}$, the AQN-coated PP membrane exhibited more than 100 times higher adsorption capacity toward anionic dyes than cationic dyes, thereby affording separation of amido black (an anionic dye) from rhodamine (a cationic dye) (Figures S27 and S28). Furthermore, the AQN-coated PP membrane showed underwater superoleophobicity, with an oil (gasoline) contact angle of $\sim 150^{\circ}$ (Figure S29), which afforded the separation of oil-in-water emulsions (e.g., toluene-in-water emulsions of $\sim 10 \mu \mathrm{m}$ in diameter) from water (Figure $5 \mathrm{~d}, \mathrm{e})$. Although the water flux decreased with operation time owing to surface fouling by toluene, it was easily recovered after rinsing with $\mathrm{EtOH}$ and water (Figure $5 f$ ), demonstrating the excellent reusability of the AQN coatings for the treatment of oil-in-water emulsions.

\section{CONCLUSION}

We have demonstrated a co-assembly methodology to prepare thin films and coatings with controllable structures and properties on a wide range of substrates. An attractive feature of the current approach is that it enables the thickness, adhesion, wettability, surface potential, and other properties of the coating to be finely 
tuned by varying the type and concentration of the building blocks used in the synthesis. The series of coatings prepared herein were used to mediate various surface-confined reactions to fabricate diverse nanostructures with controllable topology and chemistry (e.g., nanoparticles, nanorods, nanocrystals, metal-organic films). Mechanistic studies revealed that the adhesion between the building blocks and substrates was the main driving force for their assembly under water, while the Schiff base/Michael addition led to covalently cross-linked networks that were stable in a range of conditions studied (e.g., aerobic and anaerobic environments, polar and nonpolar solvents, and $\mathrm{pH}$ 1-12). We anticipate that the present study will open up avenues for generating engineered films and coatings for various applications.

\section{ACKNOWLEDGMENT}

Financial support from the National Natural Science Foundation of China (51873192 and 51522305) and Zhejiang Provincial Natural Science Foundation of China (LZ20E030002) is gratefully acknowledged. This research was also conducted and funded by the Australian Research Council Centre of Excellence in Convergent Bio-Nano Science and Technology (project number CE140100036). F.C. acknowledges the award of a National Health and Medical Research Council Senior Principal Research Fellowship (GNT1135806). R.L. acknowledges the Netherlands Organisation for Scientific Research for a Rubicon postdoctoral fellowship (project 019.182EN.034). We thank Shiyao Li for assistance with preparation of the schemes in Figure 4. This work was performed in part at the Mass Spectrometry, Proteomics Facility (MSPF), and Materials Characterisation and Fabrication Platform (MCFP) at the University of Melbourne.

\section{Conflict of interest}

The authors declare no conflict of interest.

Keywords: nanostructures $\cdot$ quinones $\bullet$ self-assembly $\bullet$ surface modification $\cdot$ thin films

[1] T. M. Schutzius, S. Jung, T. Maitra, G. Graeber, M. Köhme, D. Poulikakos, Nature 2015, 527, 82.

[2] G. Bai, D. Gao, Z. Liu, X. Zhou, J. Wang, Nature 2019, 576, 437.

[3] X. Hou, Y. Hu, A. Grinthal, M. Khan, J. Aizenberg, Nature 2015, 519, 70.

[4] J. Cai, C. Li, N. Kong, Y. Lu, G. Lin, X. Wang, Y. Yao, I. Manners, H. Qiu, Science 2019, 366, 1095.

[5] D. Y. Ryu, K. Shin, E. Drockenmuller, C. J. Hawker, T. P. Russell, Science 2005, 308, 236.

[6] B. Su, Y. Tian, L. Jiang, J. Am. Chem. Soc. 2016, 138, 1727

[7] Q. Sun, D. Wang, Y. Li, J. Zhang, S. Ye, J. Cui, L. Chen, Z. Wang, H. J. Butt, S. Vollmer, X. Deng, Nat. Mater. 2019, 18, 936.

[8] B. Liang, H. Wang, X. Shi, B. Shen, X. He, Z. A. Ghazi, N. A. Khan, H. Sin, A. M. Khattak, L. Li, Z. Tang, Nat. Chem. 2018, 10, 961.

[9] S. Karan, Z. Jiang, A. G. Livingston, Science 2015, 348, 1347.

[10] J. Niu, D. J. Lunn, A. Pusuluri, J. I. Yoo, M. A. O'Malley, S. Mitragotri, H. T. Soh, C. J. Hawker, Nat. Chem. 2017, 9, 537.

[11] R. Liu, J. Zhao, Q. Han, X. Hu, D. Wang, X. Zhang, P. Yang, Adv. Mater. 2018, 30, 1802851

[12] H. Ke, L. Wang, Z. Dai, Y. Jin, E. Qu, Z. Xing, C. Guo, X. Yue, J. Liu, Angew. Chem. Int. Ed. 2011, 50, 3017.
[13] J. C. Love, L. A. Estroff, J. K. Kriebel, R. G. Nuzzo, G. M. Whitesides, Chem. Rev. 2005, 105, 1103

[14] T. Ozel, B. A. Zhang, R. Gao, R. W. Day, C. M. Lieber, D. G. Nocera, Nano Lett. 2017, 17, 4502.

[15] F. Caruso, R. A. Caruso, H. Möhwald, Science 1998, 282, 1111

[16] G. Decher, Science 1997, 277, 1232.

[17] J. J. Richardson, M. Björnmalm, F. Caruso, Science 2015, 348, aaa2491

[18] H. Lee, S. M. Dellatore, W. M. Miller, P. B. Messersmith, Science 2007, 318,426

[19] H. Ejima, J. J. Richardson, K. Liang, J. P. Best, M. P. van Koeverden, G. K. Such, J. Cui, F. Caruso, Science 2013, 341, 154

[20] H. Lee, B. P. Lee, P. B. Messersmith, Nature 2007, 448, 338.

[21] Q. Ye, F. Zhou, W. Liu, Chem. Soc. Rev. 2011, 40, 4244.

[22] C. Zhang, Y. Ou, W. X. Lei, L. S. Wan, J. Ji, Z. K. Xu, Angew. Chem. Int. Ed. 2016, 55, 3054

[23] M. Shin, H. A. Lee, M. Lee, Y. Shin, J. J. Song, S.W. Kang, D. H. Nam E. J. Jeon, M. Cho, M. Do, S. Park, M. S. Lee, J.-H. Jang, S.W. Cho, K. S. Kim, H. Lee, Nat. Biomed. Eng. 2018, 2, 304.

[24] Z. Wang, H. C. Yang, F. He, S. Peng, Y. Li, L. Shao, S. B. Darling, Matter 2019, 1, 115.

[25] J. Guo, B. L. Tardy, A. J. Christofferson, Y. Dai, J. J. Richardson, W. Zhu, M, Hu, Y. Ju, J. Cui, R. R. Dagastine, I. Yarovsky, F. Caruso, Nat. Nanotechnol. 2016, 11, 1105.

[26] A. S. Goldmann, N. R. B. Bose, L. Michalek, J. P. Blinco, A. Welle, and C. Barner-Kowollik, Adv. Mater. 2019, 31, 1902665.

[27] J. Li, A. D. Celiz, J. Yang, Q. Yang, I. Wamala, W. Whyte, B. R. Seo, N. V. Vasilyev, J. J. Vlassak, Z. Suo, D. J. Mooney, Science 2017, 357, 378.

[28] H. Yuk, C. E. Varela, C. S. Nabzdyk, X. Mao, R. F. Padera, E. T. Roche, X. Zhao, Nature 2019, 575, 169

[29] A. H. Hofman, I. A. van Hees, J. Yang, M. Kamperman, Adv. Mater 2018, 30, 1704640

[30] Q. Zhao, D. W. Lee, B. K. Ahn, S. Seo, Y. Kaufman, J. N. Israelachvili, J. H. Waite, Nat. Mater. 2016, 15, 407.

[31] S. Singla, G. Amarpuri, N. Dhopatkar, T. A. Blackledge, A. Dhinojwala, Nat. Commun. 2018, 9, 1890

[32] Q. Lu, D. X. Oh, Y. Lee, Y. Jho, D. S. Hwang, H. Zeng, Angew. Chem Int. Ed. 2013, 52, 3944.

[33] H. Yu, Q.-Z. Zhong, T. G. Liu, W. Z. Qu, B. H. Wu, Z. K. Xu, L. S. Wan, Langmuir 2019, 35, 3643

[34] J. Zheng, R. Fan, H. Wu, H. Yao, Y. Yan, J. Liu, L. Ran, Z. Sun, L. Yi, L. Dang, P. Gan, P. Zheng, T. Yang, Y. Zhang, T. Tang, Y. Wang, Nat. Commun. 2019, 10, 1604

[35] S. E. Rossiter, M. H. Fletcher, W. M. Wuest, Chem. Rev. 2017, 117, 12415.

[36] M. G. Peter, Angew. Chem. Int. Ed. 1989, 28, 555.

[37] P. J. J. Alvarez, C. K. Chan, M. Elimelech, N. J. Halas, D Villagrán, Nat. Nanotechnol. 2018, 13, 634

[38] M. W. Tibbitt, J. E. Dahlman, R. Langer, J. Am. Chem. Soc. 2016, 138 704.

[39] B. Piro, J. Haccoun, M. C. Pham, L. D. Tran, A. Rubin, H. Perrot, C. Gabrielli, J. Electroanal. Chem. 2005, 577, 155.

[40] S. Guo, X. Zhu, D. Jańczewski, S. S. C. Lee, T. He, S. L. M. Teo, G. J. Vancso, Nat. Nanotechnol. 2016, 11, 817

[41] C. Lim, J. Huang, S. Kim, H. Lee, H. Zeng, D. S. Hwang, Angew. Chem Int. Ed. 2016, 55, 3342; Angew. Chem. 2016, 128, 3403.

[42] G. Meng, T. Guo, T. Ma, J. Zhang, Y. Shen, K. B. Sharpless, J. Dong, Nature 2019, 574, 86

[43] T. Hasell, A. I. Cooper, Nat. Rev. Mater. 2016, 1, 16053.

[44] Y. Xu, S. Jin, H. Xu, A. Nagai, D. Jiang, Chem. Soc. Rev. 2013, 42, 8012.

[45] O. Shekhah, J. Liu, R. A. Fischerb, C. Wöll, Chem. Soc. Rev. 2011, 40, 1081.

[46] S. Salunke-Gawali, E. Pereira, U. A. Dar, S. Bhand, J. Mol. Struct. 2017, $1148,435$.

[47] F. Rothman, J. Org. Chem. 1958, 23, 1049

[48] Q.-Z. Zhong, J. J. Richardson, S. Li, W. Zhang, Y. Ju, J. Li, S. Pan, J. Chen, F. Caruso, Angew. Chem. Int. Ed. 2020, 59, 1711; Angew. Chem. 2020, 132, 1728. 
[49] H. Wang, P. Hu, J. Yang, G. Gong, L. Guo, X. Chen, Adv. Mater. 2015, 27, 2348.

[50] J. A. Berenbeim, S. Boldissar, S. Owens, M. R. Haggmark, G. Gate, F. M. Siouri, T. Cohen, M. F. Rode, C. Schmidt Patterson, M. S. de Vries, Sci. Adv. 2019, 5, eaaw5227.

[51] Y. Liang, Y. Jing, S. Gheytani, K. Y. Lee, P. Liu, A. Facchetti, Y. Yao, Nat. Mater. 2017, 16, 841.

[52] K. Lin, Q. Chen, M. R. Gerhardt, L. Tong, S. B. Kim, L. Eisenach, A. W. Valle, D. Hardee, R. G. Gordon, M. J. Aziz, M. P. Marshak, Science 2015, 349, 1529 . 


\section{Entry for the Table of Contents}

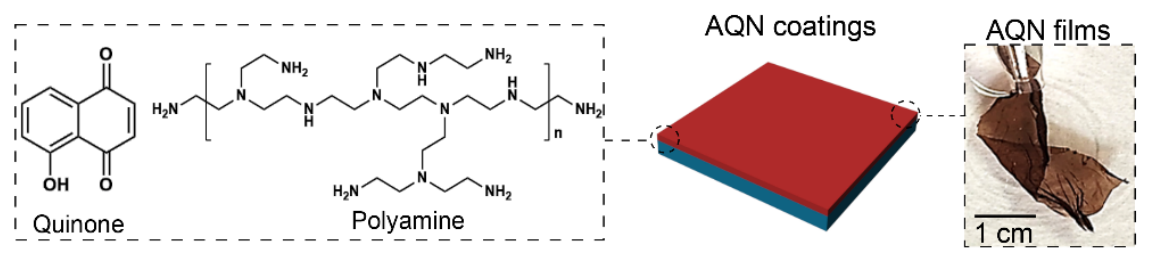

Coatings and thin films of amino-quinone networks are engineered on various materials by a co-assembly strategy using quinones and polyamines. Employing different building blocks affords precise control over the structures and properties of the coatings, which can also serve as multifunctional reaction platforms to synthesize diverse nanoparticles on surfaces. 


\section{University Library}

\section{- M M N E R VA A gateway to Melbourne's research publications}

Minerva Access is the Institutional Repository of The University of Melbourne

Author/s:

Zhong, Q-Z;Richardson, JJ;He, A;Zheng, T;Lafleur, RPM;Li, J;Qiu, W-Z;Furtado, D;Pan, S;Xu, Z-K;Wan, L-S;Caruso, F

Title:

Engineered Coatings via the Assembly of Amino-Quinone Networks

Date:

2020-11-30

\section{Citation:}

Zhong, Q. -Z., Richardson, J. J., He, A., Zheng, T., Lafleur, R. P. M., Li, J., Qiu, W. -Z., Furtado, D., Pan, S., Xu, Z. -K., Wan, L. -S. \& Caruso, F. (2020). Engineered Coatings via the Assembly of Amino-Quinone Networks. ANGEWANDTE CHEMIE-INTERNATIONAL EDITION, 60 (5), pp.2346-2354. https://doi.org/10.1002/anie.202010931.

Persistent Link:

http://hdl.handle.net/11343/253881 